\title{
Alternate Marking-based Network Telemetry for Industrial WSNs
}

\author{
Abdulkadir Karaagac, Eli De Poorter, Jeroen Hoebeke \\ Ghent University - imec, IDLab, Department of Information Technology \\ Technologiepark Zwijnaarde 126, B-9052 Ghent, Belgium \\ \{abdulkadir.karaagac, eli.depoorter, jeroen.hoebeke\}@ugent.be
}

\begin{abstract}
For continuous, persistent and problem-free operation of Industrial Wireless Sensor Networks (IWSN), it is critical to have visibility and awareness into what is happening on the network at any one time. Especially, for the use cases with strong needs for deterministic and real-time network services with latency and reliability guarantees, it is vital to monitor network devices continuously to guarantee their functioning, detect and isolate relevant problems and verify if all system requirements are being met simultaneously. In this context, this article investigates a light-weight telemetry solution for IWSNs, which enables the collection of accurate and continuous flowbased telemetry information, while adding no overhead on the monitored packets. The proposed monitoring solution adopts the recent Alternate Marking Performance Monitoring (AMPM) concept and mainly targets measuring end-to-end and hopby-hop reliability and delay performance in critical application flows. Besides, the technical capabilities and characteristics of the proposed solution are evaluated via a real-life implementation and practical experiments, validating its suitability for IWSNs.

Index Terms-AM-PM, Network Telemetry, Network Monitoring, WirelessHart, ISA100.11a, IEEE 802.15.4e TSCH, 6TiSCH.
\end{abstract}

\section{INTRODUCTION}

Despite their success in industrial monitoring applications, Industrial Wireless Sensor Network (IWSN) technologies, such as WirelessHart [1], ISA100.11a [2], and finally 802.15.4e TSCH [3] and 6TiSCH (IPv6 over the TSCH mode of IEEE 802.15.4e) [4], are facing significant challenges in supporting the use cases with strong needs for deterministic and real-time network services with latency and reliability guarantees; such as real-time control systems.

In this sense, one of the main obstacles for achieving deterministic and time-critical connectivity in IWSNs is the insufficiency of existing network monitoring mechanisms for advanced network management operations. Currently, these IWSN technologies only define monitoring operations based on periodical or event-driven information or alarm reports from field devices, typically regarding node state or connectivity such as health reports and link/neighbor statistics [5]. These techniques offer limited capabilities for collecting detailed and timely in-network state and performance information and verifying services for critical application traffic. Therefore, there is a need for continuous and precise monitoring mechanisms for IWSNs to monitor critical applications, guarantee their functioning, detect and isolate relevant problems and verify if all system requirements are being met simultaneously.
In the last few years, Network Telemetry became a prominent trend in the networking community by enabling accurate measurement of the network's performance in real-time [6]. Especially, as an alternative to the traditional monitoring techniques, recently introduced In-Band Network Telemetry (INT) approaches allow fine-grained per-hop and per-packet measurements to be collected as packets traverse a network [7]. However, these approaches require on-the-fly packet alteration and introduce extra per-packet overhead on the application packets which may not be preferable for time-critical traffic flows. On the other hand, Alternate Marking Performance Measurement (AM-PM) is another recent, reliable and efficient measurement method for monitoring network flows (loss and delay measurements) with low overhead, namely at the cost of one or two bits per data packet [8]. Currently, this technique is under development in the Internet Engineering Task Force (IETF) and an RFC is already published regarding the concept and methodology [9].

In this context, the main goal of this work is the design of a light-weight and efficient monitoring solution for IWSN technologies, which will enable precise performance monitoring for network flows, especially critical application traffic. For this purpose, the recently proposed concept of AM-PM is adopted and deployed over the 6TiSCH Network stack, by using an existing unused bit in the 802.15.4 MAC Header; thus, without any overhead or impact on the monitored application traffic. The proposed monitoring solution creates an accurate flow-based telemetry solution for measuring end-to-end and hop-by-hop reliability and delay performance, which yields various monitoring and management functionalities in IWSNs, including service verification and problem troubleshooting.

The main contributions of this paper are as follows:

- The first, to the best of found knowledge, conceptual design for an AM-PM telemetry mechanism adapted to WSNs.

- A flexible and adaptive network monitoring mechanism, with minimal overhead, for IEEE 802.15.4e TSCH Networks, which was inspired by AM-PM.

- The first, to the best of found knowledge, realization and deployment of AM-PM method on a multihop network with full loss and delay measurement functionalities.

- The implementation of the proposed design and end-toend validation and evaluation of the proposed architecture via a real-life 6TiSCH Network with the AM-PM ability. 
The remainder of the paper is organized as follows. Section II introduces relevant concepts and provides technical background about the target technologies. After that, the proposed AM-PM network monitoring mechanism for IWSNs is presented in Section III. Next, Section IV presents an evaluation and validation study about the proposed solution via theoretical and practical experiments, which is followed by a detailed discussion about the monitoring capabilities of AM-PM in IWSNs in Section V. Finally, Section VI concludes the paper.

\section{TECHNICAL BACKGROUND}

\section{A. Industrial Wireless Sensor Networks}

Over the past decade, we have seen the emergence of several IWSN technologies based on a Time-Slotted Channel-Hopping (TSCH) scheme to meet the stringent performance requirements of industrial applications, including WirelessHART, ISA100.11a and finally IEEE 802.15.4e TSCH and 6TiSCH. Thanks to the pre-allocated communication resources and the channel hopping mechanism, these TSCH-based wireless networks are able to yield extremely high end-to-end reliability and support for deterministic communication scheme while ensuring over a decade of battery lifetime [10].

In a TSCH network, time is globally synchronized and sliced up into time slots, and the overall communication is orchestrated by a schedule that defines the action (transmit, receive, sleep) of each node in each time slot [3]. In this TSCH schedule, a single element, named cell, is identified by a pair of slotOffset and channelOffset, which is used to define the communication time and frequency. The proper functioning of a TSCH network depends on this schedule which can be typically created in various ways, but should be computed according to the specific requirements of the applications, such as latency, reliability, and energy. The time synchronization in a TSCH network means that all nodes share a timeslot counter (encoded using 5 bytes in IEEE 802.15.4e), typically named Absolute Slot Number (ASN), for the total number of slots that have passed since the network has started [3]. An architectural overview of these TSCH-based Industrial WSNs technologies and their communication schemes are presented in Fig. 1.

Among the TSCH-based technologies, IEEE 802.15.4e is a recent MAC amendment of the IEEE 802.15.4 standard, specially designed for harsh industrial environments with a reliable and deterministic communication scheme [3]. The more recently proposed $6 \mathrm{TiSCH}$ architecture binds the prior IPv6-enabled standards (6LoWPAN, RPL, CoAP) with IEEE 802.15.4e TSCH and defines a standardized approach to build and maintain a schedule. Compared to its TSCH-based predecessors, 6TiSCH has opted for an open and standardized communication stack as well as support for different scheduling schemes, turning it into a more open and flexible, but also equally reliable and deterministic wireless communication solution. 6TiSCH also defines directed and deterministic paths, named Tracks, between source and destination nodes across a network, in order to create guarantees of end-to-end latency and jitter, especially targeting time-critical applications [11].

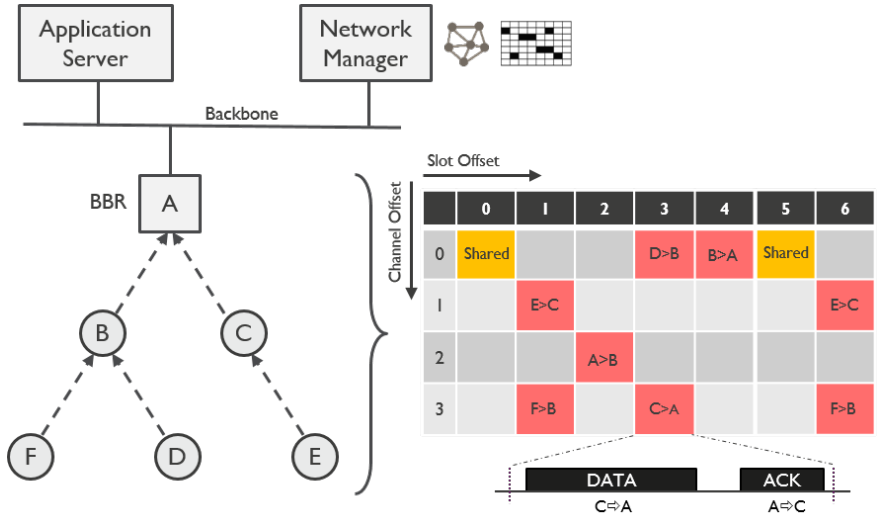

Fig. 1: Architecture and schedules in TSCH-based Industrial WSNs.

\section{B. Network Telemetry and Monitoring for Industrial WSNs}

Typically, IWSN technologies employ centralized management operations (e.g. routing, scheduling) for achieving better bandwidth occupation and more reliable and deterministic behavior. For instance, in WirelessHART and ISA100.11a, the central network manager regulates all the communication in the network, while 6TiSCH allows centralized network and schedule management mechanisms along with distributed and mixed schemes [12]. Regardless of the technology, any of the centralized management tasks requires establishing and maintaining a network image continuously at a central entity, generally named the Network Manager (NM), to be processed and analyzed often in real-time.

Therefore, as presented in Fig. 2, Network Monitoring and Telemetry is an ecologically vital mechanism for IWSNs to achieve a wide range of management operations, including anomaly detection, traffic engineering (schedules, routes), network optimization and service verification. In WirelessHART and ISA100.11a, the network management fully relies on periodical or event-driven information or alarm reports from field devices to the Network Manager [1] [2]. These measurement data can typically be node or network state information such as health/failure reports, link/neighbor statistics, network topology and node/link occupancy [5]. While, 6TiSCH defines a management interface, based on CoAP Management Interface (CoMI) [13], which can be used to monitor network performance and perform network configurations.

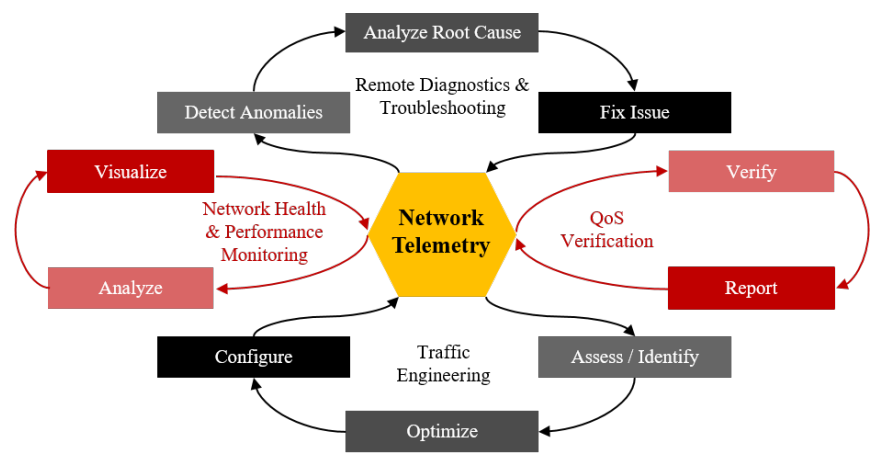

Fig. 2: The role of the Network Telemetry for IWSNs. 
However, the scope of all these diagnosis and monitoring solutions is quite limited in terms of capability, efficiency and flexibility considering the required functionalities for advanced network management operations for IWSNs. These tools are not sufficient, particularly for monitoring flow and packetbased performance, detecting and reporting transient network congestion, and isolating fault location.

In this regard, In-band Network Telemetry (INT) mechanism, also referred to as In-situ Operations, Administration, and Maintenance (iOAM) [14], is recently proposed as an alternative to these traditional monitoring techniques for Industrial WSNs [15]. As presented in [15], INT can offer an ultra-efficient network monitoring solution with minimized communication overhead while supporting a wide range of monitoring operations and strategies for dealing with various network scenarios and use cases. However, this approach only collects telemetry data about the delivered packets and does not provide any information about the lost packets, which yields a limitation for reliability measurements. Moreover, it relies on the idea of processing and altering monitored packets in each hop, which may potentially impact the forwarding behavior of the packets. Additionally, the piggybacking method results in adding telemetry entries to the packets, which yields, limited but still, extra overhead on the monitored packets. These aforementioned features of INT mechanism might be an issue, especially in time-critical deterministic traffic flows.

Therefore, there is a need for a flexible, efficient and accurate network monitoring solution for IWSNs which can complement existing monitoring and telemetry mechanisms by providing a per-hop and per-flow telemetry solution with zero effect and overhead on the monitored packets and enabling automated checks on the network to ensure that it is performing as intended, especially for critical application traffic.

\section{AM-PM Overview}

Alternate Marking is a monitoring method that was first introduced in 2011, primarily targeting packet loss measurement [16]. After years of effort, it is turned into an RFC as a method to perform packet loss, delay, and jitter measurements on live traffic [9]. And currently, AM-PM is under development in the IETF in the context of various encapsulation protocols, including MPLS, QUIC and Geneve [17].

In AM-PM, every packet of the monitored flow carries one or two marking bits that are used for signaling and coordinating measurement events across the measurement points. For packet loss measurements, a periodically alternating marker, Color Bit, is dividing the traffic into consecutive blocks of data. By counting the number of packets in each block and comparing the values measured by different network devices along the path, it is possible to measure packet loss occurred in any single block between any two points [9]. While for delay measurements, another marker, Delay Bit, is used to notify a network device to store the timestamps for the particular packets. By comparing these timestamps with timestamps of the same packets on other measurement points, delay values for each packet on each hop can be computed.
However, since measurement points only have their own measurement samples, they are not able to directly use the stored information to measure packet loss or delay. For this reason, an external Network Management Entity collects and elaborates telemetry data to calculate the end-to-end and hopby-hop performance. This means that the AM-PM mechanism requires additional control traffic for collecting the telemetry data from each node on the path of the monitored flow.

According to [9], the color and delay measurement indicators can be simply transferred with two separate bits, which results in Double Marking method. However, this information can be also signaled with a single bit representing both information via several methods (multiplexing, hashing, etc.), presenting a trade-off in terms of factors such as implementation simplicity or resilience to out-of-order delivery [18].

Although AM-PM is not yet a widely used industry standard, it has already been applied to a number of nonconstrained networking platforms. For instance, a variant of AM-PM is already implemented in commodity network devices by Huawei, which was called Packet Conservation Algorithm for Internet (iPCA) [19]. Similarly, Telecom Italia has been using and testing the AM-PM methods in very large deployments with cellular networks [17]. Additionally, [8] presents a detailed implementation approach of AM-PM that was applied to one hardware and one software-based implementation along with experimental evaluation results.

\section{AM-PM FOR 6TISCH NETWORKS}

As for any IWSN technology, network monitoring is a vital mechanism for $6 \mathrm{TiSCH}$, where nodes are continuously or periodically monitored to perform various network management operations. Especially, for time-critical applications, it is required for 6TiSCH networks to collect and analyze telemetry data to ensure that it is performing as intended.

This section presents the design of a light-weight and accurate monitoring solution, mainly based on the AM-PM mechanism, for 6TiSCH networks with zero effect and overhead on the monitored packets. The high-level overview of the multi-point measurement architecture for AM-PM in IWSNs is presented in Fig. 3.

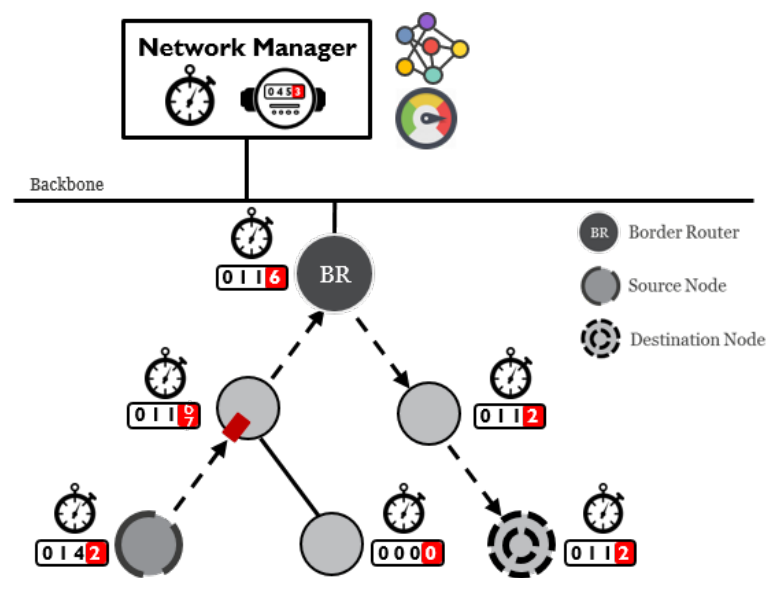

Fig. 3: AM-PM in 6TiSCH Network Architecture. 
As illustrated in Fig. 3, the telemetry data is collected on each hop while a packet from the monitored flow is traversing over the network. When the end of the monitoring period is reached, the telemetry reports are generated, to be used by the Network Manager (NM) for further processing and analysis. By means of the proposed solution, it is possible to obtain end-to-end packet and flow level network information (e.g. latency, reliability). In addition, it also enables hop-byhop telemetry that can provide per-hop reliability, latency and jitter performances, which can be used to detect which rules a packet followed and to detect how long a monitored packet was queued on each node.

\section{A. System Design}

As described in Section II-C, AM-PM relies on the idea that every packet of the monitored flow carries one or two marking bits (Color and Delay Bits) for signaling and coordinating the measurement between the measurement nodes. In the design of AM-PM for 6TiSCH, the multiplexed marking scheme is used, where the Color and Delay Bits are multiplexed (exclusive or XOR) into a single bit. And for this marking bit, as shown in Fig. 4 , the existing unused bit ( $7^{\text {th }}$ bit) in the Frame Control field of the IEEE 802.15.4 header is used as the dedicated AM-PM marking bit [20]. Therefore, the proposed solution brings no extra overhead on the monitored packet.

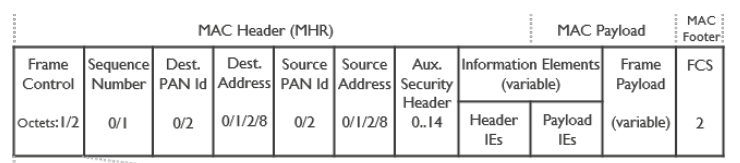

\begin{tabular}{|c|c|c|c|c|c|c|c|c|c|c|}
\hline $0-2$ & 3 & 4 & 5 & 6 & 7 & 8 & 9 & $10-11$ & $12-13$ & $14-15$ \\
\hline $\begin{array}{c}\text { Frame } \\
\text { Type }\end{array}$ & $\begin{array}{c}\text { Security } \\
\text { Enabled }\end{array}$ & $\begin{array}{c}\text { Frame } \\
\text { Pending }\end{array}$ & $\begin{array}{c}\text { ACK } \\
\text { Request }\end{array}$ & $\begin{array}{c}\text { PAN ID } \\
\text { Comp. }\end{array}$ & $\begin{array}{c}\text { AM-PM } \\
\text { (Reserved) }\end{array}$ & $\begin{array}{c}\text { Sequence } \\
\text { No. Supp. }\end{array}$ & $\begin{array}{c}\text { IE } \\
\text { Present }\end{array}$ & $\begin{array}{c}\text { Destination } \\
\text { Addr. Mode }\end{array}$ & $\begin{array}{c}\text { Frame } \\
\text { Version }\end{array}$ & $\begin{array}{c}\text { Source } \\
\text { Addr. Mode }\end{array}$ \\
\hline
\end{tabular}

Fig. 4: IEEE 802.15.4 Frame Format and AM-PM Bit.

For detecting the Color and Delay Bits, a mechanism is introduced as a function of the ASN: the $k^{\text {th }}$ least significant bit (LSB) of ASN is used as the Color Bit (packet loss measurements), while the rising edge of the $(k-1)^{t h}$ bit of ASN is used for determining the Delay Bit (delay measurements). Therefore, for delay measurements, the delay bit is assigned a pulse value one per time interval which indicates that the marked packet is a reference for the measurement, i.e. capture the timestamp of this packet.

As shown in Fig. 5, this ASN-based marking mechanism divides time into a collection of slots which results in fixed time intervals between the toggling of the bits, thus between telemetry operations. The measurement frequency fully depends on the chosen $k$ value. A smaller $k$ value will increase the measurement frequency, thereby allowing fine-grained measurement with a cost of larger management overhead. So, the measurement granularity and management overhead are strictly dependent.

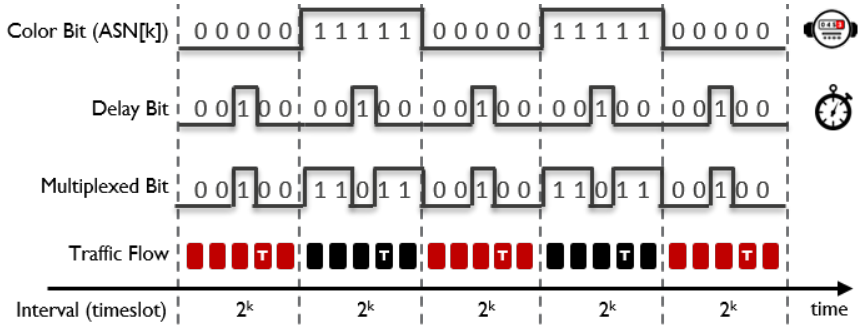

Fig. 5: Single-bit ASN-colored Multiplexed Marking.

For packet loss measurements, on each measuring hop, two counters are maintained, one per color, for each flow. At the end of each interval, the particular counter is reported to NM and analyzed. By using the reports from all the nodes on the path, NM can perform accurate loss measurements and detect the lossy link/hop in case of packet losses. While for delay measurements, only one of the packets is marked in each interval for delay measurement which results in the flipping of the value of the multiplexed bit compared to the other packets in the same block. A pulse detection operation allows other measurement hops to identify the timestamping signals (Delay Bit) on the designated packets. Then, thanks to the synchronized ASN, one-way-delay between two measurement points can be computed by simply comparing two timestamps measured in the same interval.

For distinguishing the monitored traffic flows in $6 \mathrm{TiSCH}$ Networks, the 3-tuple of the Flow Label, Source Address, and Destination Address fields in the IPv6 header is used.

\section{B. Measurement Process}

The measurement for a flow starts on the first time the Color Bit is set to 1. From now on, all the nodes on the path of the monitored flow will count the number of packets for a given color in the corresponding monitoring interval identified by the Color Bit. Throughout a monitoring interval for a certain color, if the monitoring nodes detect a color change that only lasted a single packet, they fire a timestamp event and record the current ASN to be reported at the end of the period.

On the other hand, whenever a predefined number, represented as $n$, of consecutive packets with the other color is received, the node fires a color change and creates a reporting message for the finalized color, including the packet counter and the timestamp. The value of $n$ should be chosen large enough to prevent any kind of measurement inaccuracies due to out-of-order delivery and dropped packets. However, very large $n$ values will result in the delay of the telemetry reports. Alternatively, thanks to the synchronized ASN across the network, the border router can detect the color change and collect counters/telemetry data safely upon color changes.

Lastly, if the value of the Color Bit does not change for the packets from three consecutive intervals, the measurements for the particular flow will be discarded and terminated.

\section{The cost of the AM-PM operation}

Since it harnesses an existing bit in the IEEE 802.15.4 header, the proposed AM-PM mechanism brings no extra 
overhead on the monitored packets. Besides, there is a limited amount of extra resource usage by the source, destination and forwarding nodes of the monitored traffic for reporting the collected telemetry data. However, this extra resource can be eliminated or minimized if it is combined with other health and status reporting messages in the technology like WirelessHART and ISA100.11a. While, for 6TiSCH, the nodes can be strained to use shared resources and avoid resources dedicated to critical communication, which will prevent any effect on the network performance for the critical applications. In terms of memory, two counters and one timestamp for every monitored flow is needed on each device on the monitored traffic path. While, due to extremely low computational effort, the additional load on processing is quite negligible.

\section{Implementation}

In order to validate and demonstrate the proposed telemetry and monitoring solution, we implemented its fundamental functionalities in a widely used operating system for embedded IoT and wireless sensor devices with IPv6 connectivity: Contiki $N G$ [21]. Contiki-NG is an open-source, cross-platform operating system for IoT devices and it focuses on dependable IoT and low-power communication protocols such as 6LoWPAN, RPL, and CoAP [21]. Recently, it has also been extended with support for TSCH and 6TiSCH with so-called "minimal configuration" [22] along with a simple Scheduling Function (SF) (sf-simple) which provides APIs for user processes in order to add or remove cells dynamically.

In order to achieve AM-PM, the 6TiSCH protocol implementation is extended so that nodes can process AM-PM events for monitored application flows and periodically report the collected networking performance. Fig. 6 illustrates the 6 TiSCH Network stack complemented with AM-PM crosslayer facilities. First of all, this cross-layer agent cooperates with Network and MAC layer in order to retrieve telemetry data (timestamps) and relevant attributes (marking bits, flow label, etc.) about the processed packet. For every generated, forwarded and received packet for monitored flows, the AMPM agent needs to maintain two counters.

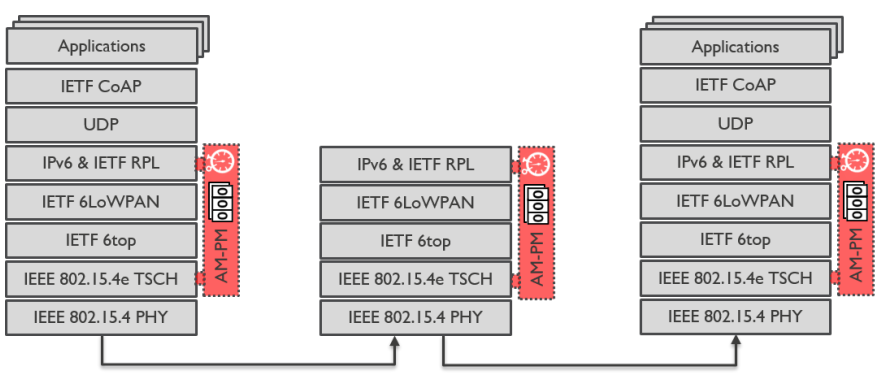

Fig. 6: 6 TiSCH Network Stack with AM-PM Cross-Layer Agent.

In addition to the AM-PM extensions to the 6TiSCH stack, a Network Monitoring Application is also implemented which is used to collect and analyze AM-PM telemetry data and extract insights about the network performance in order to monitor packet loss rate, latency and jitter performance at each hop.

\section{Evaluation AND VALIDATION}

First, this section presents an evaluation and validation study of the proposed telemetry solution in a simulation environment. Next, the results of testbed experiments are presented in order to demonstrate the operation of the AM-PM mechanism in real-world networks.

\section{A. Packet Loss and Delay Measurements via AM-PM}

In order to investigate the applicability of the AM-PM telemetry in Industrial WSNs, a series of tests are performed by using Cooja, a simulation platform for wireless sensor network applications with Contiki motes. We first run a network with application traffic (from mote 4 to mote 6 , random delays $[0.5,1.1$ seconds]) that the network can handle without any congestion and packet drops. Then, the same network is simulated in case of a data generation rate at a level (from mote 4 to mote 6 , random delays [0.1, 1.1 seconds]) that the network can no longer handle, thus yielding temporary congestion and packet losses.

For the TSCH schedule, the sf-simple scheduling function is used which schedules each node with a single outgoing cell towards the parent and a maximum of six cells in total including the incoming cells from each RPL child. For AMPM marking, the $12^{\text {th }}$ LSB of the ASN is used as Color Bit, while the rising edge of the $11^{\text {th }}$ LSB is used for generating pulse-based Delay Bit. That means the Color Bit and Delay Bit were toggled approximately every 20 seconds which resulted in periodical (20 seconds) telemetry reports from monitoring motes to the Border Router (BR) destined to the Network Manager. The simulation setup and detailed network parameters and application settings are provided in Fig. 7.

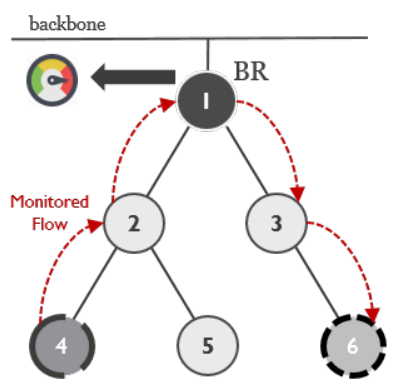

\begin{tabular}{ll}
\hline Parameter & Value \\
\hline \# of Sensor Motes & $5+1$ BBR \\
Data Generation & $0.1-1.1$ second \\
Time Slot Duration & $10 \mathrm{~ms}$ \\
Length of Slotframe & 11 \\
802.15.4 Channels & {$[15,20,25,26]$} \\
Queue Size & 4 \\
AM-PM - Color Bit & $12^{\text {th }}$ LSB of ASN \\
AM-PM - Delay Bit & $11^{\text {th }}$ LSB of ASN \\
Duration & 25 minutes \\
\hline
\end{tabular}

Fig. 7: Simulation setup with detailed settings.

Using the given setup, we performed end-to-end and hopby-hop measurements on the packet loss rate and delay for all scenarios. The results of these measurements are presented in Fig. 8. In Fig. 8a and Fig. 8b, the per-hop packet loss rate measurements are provided in case of manageable and excessive application traffic, respectively. While in Fig. 8c and Fig. 8d, the delay measurements (in terms of timeslots) are provided with a detailed representation of the actual delay on each hop. Finally, Fig. 8e and Fig. 8f show the share of each hop in the total end-to-end delay of the packets, which helps 


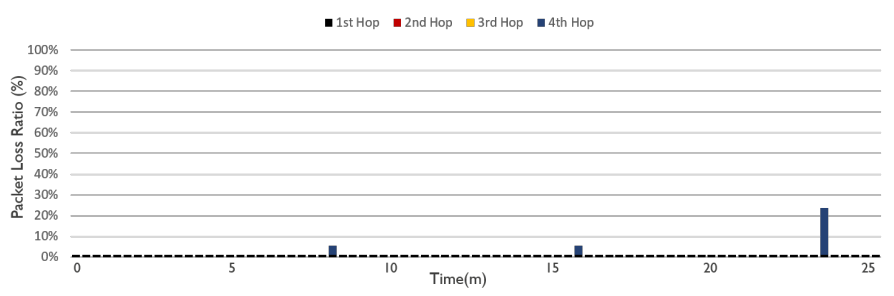

(a) Packet Loss Rate - Manageable Traffic.

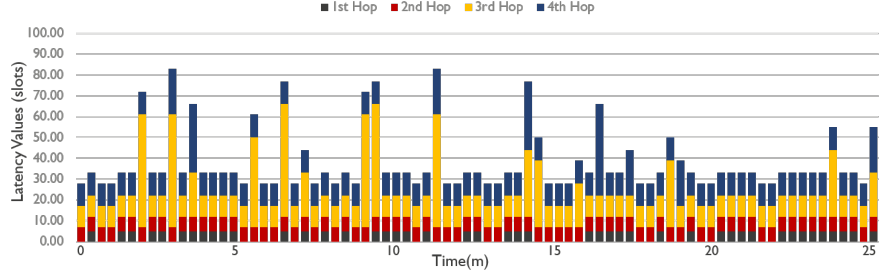

(c) Per-Hop Latency Values - Manageable Traffic.

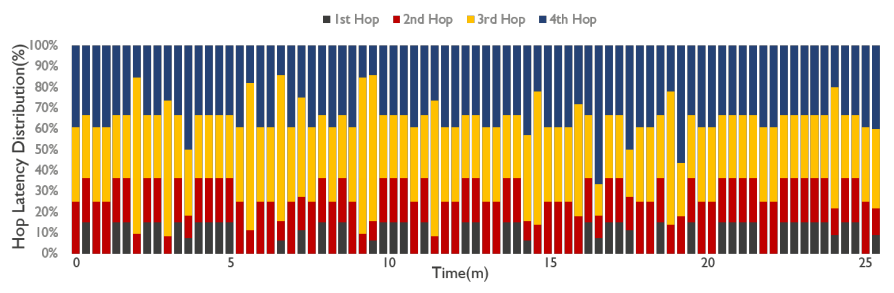

(e) The Share of Each Hop in Total Latency - Manageable Traffic.

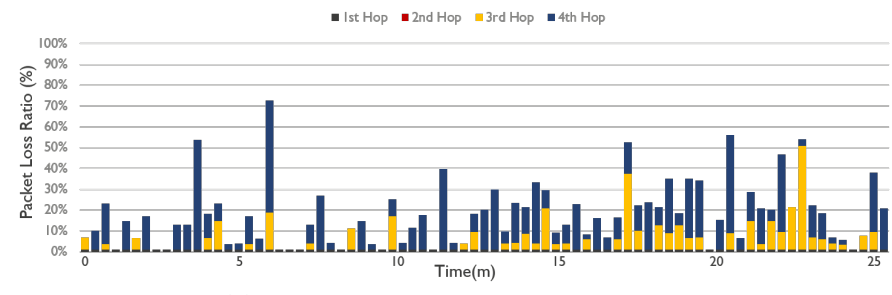

(b) Packet Loss Rate - Excessive Traffic.

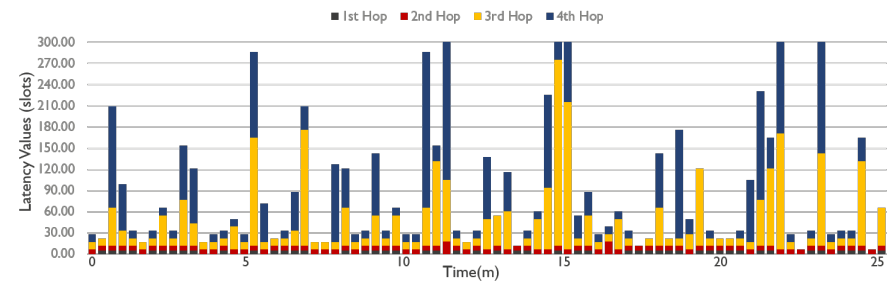

(d) Per-Hop Latency Values- Excessive Traffic.

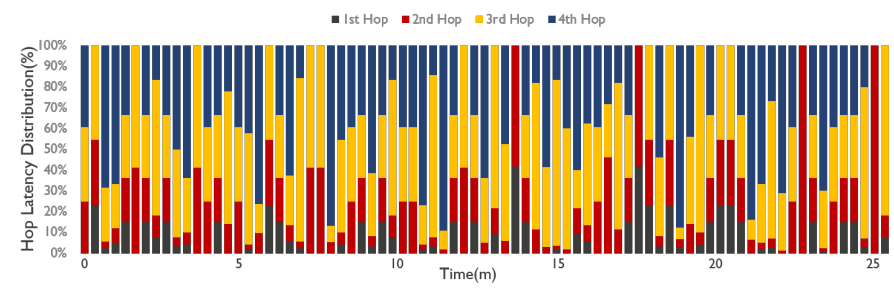

(f) The Share of Each Hop in Total Latency - Excessive Traffic.

Fig. 8: Per-Packet and Per-Hop Packet Loss Rate and Latency Measurement Results.

to understand and determine which node or hop is the main reason for a poor delay performance.

These figures present that when the monitored application traffic has a manageable data rate, the network can provide adequate service with near-zero packet loss rate and stable delay performance. While, in case of excessive traffic, the network starts to suffer from higher packet loss rates (due to congestion and buffer overflow), and also larger and unstable end-to-end latency values (due to larger queuing time).

\section{B. Testbed Evaluation}

In this subsection, the results of basic testbed experiments are presented that were conducted in order to validate and evaluate the AM-PM mechanism in real Industrial WSNs. For this purpose, several tests are performed in various scenarios in two wireless testbeds, named the Wireless Testlab (w-iLab.2) and the OfficeLab (w-iLab.1), which offer several industry or office-like wireless settings [23]. The utilized testbed consists of Zolertia Remote nodes that are static and do not move. A sample experimental setup and typical network topologies are presented in Fig. 9. In these experiments, the AM-PM-enabled 6TiSCH stack implementation in Contiki NG is used, along with available basic RPL multihop routing protocol.

After the network has been constructed and the critical application traffic initiated, AM-PM telemetry information is gathered at BR and delivered to a monitoring application where the data is stored and processed. Then, the telemetry data is mainly analyzed to understand end-to-end and hop-byhop loss rate and delay performance of the monitored critical application traffic. Some sample measurements are presented in Fig. 10, Fig. 11 and Fig. 12.

In these experiments, we performed measurements in a 3phase scenario. In the initial phase, the performance monitoring is performed in case of critical traffic flowing from Node 4 to Node 6 at a rate (random delays [0.2 - 1.2 seconds]) that can be handled by the network. Then, in phase 2 , an extra noncritical, thus not monitored, traffic from Node 5 and 2 towards BR is initiated with the same data generation rate. Finally, in the last phase, the traffic rate of the monitored application traffic from Node 4 is slightly increased to a rate (random delays [0.1 - 1.1 seconds]) where the network cannot handle it anymore. The corresponding network performances in each phase are presented in Fig. 10 separately. In addition, the average and maximum latency and packet loss rate values obtained during these experiments are provided in Fig. 11 and 12, for End-to-End and Hop-by-Hop measurements respectively.
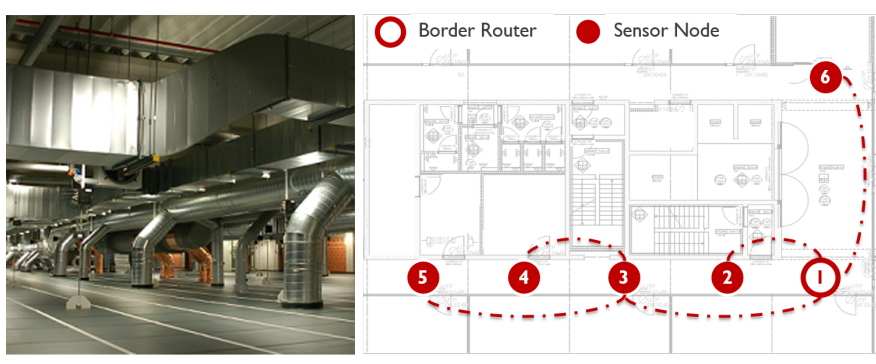

Fig. 9: Testbed experimental setup \& network topologies. 


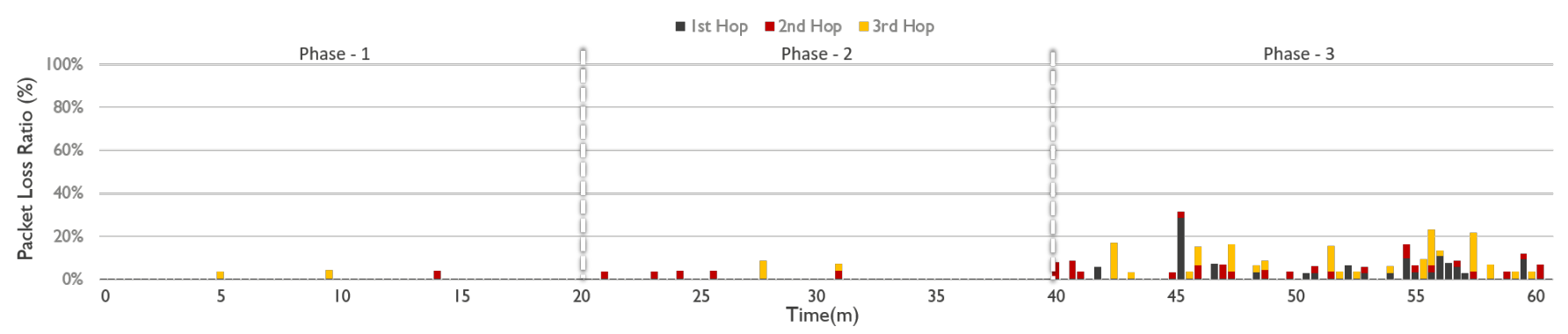

(a) Packet Loss Rate.

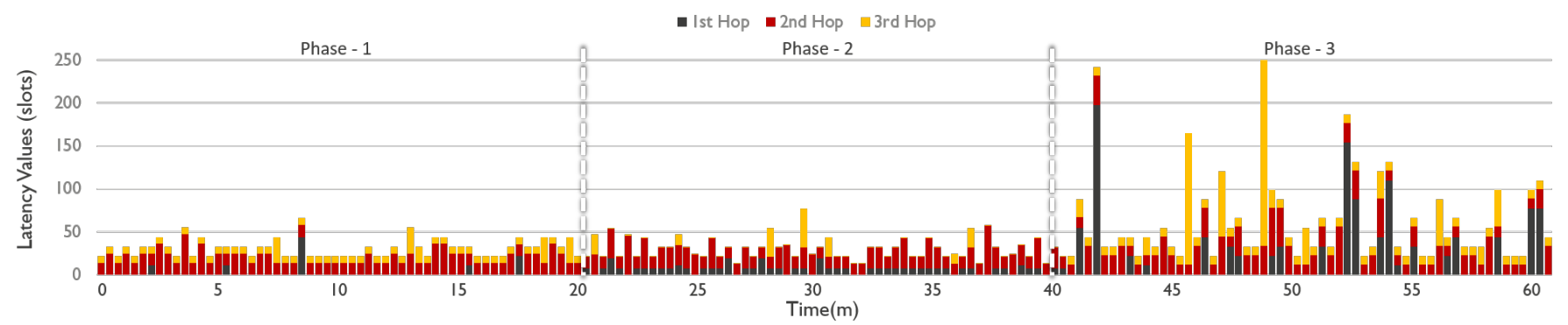

(b) Per-Hop Latency Values.

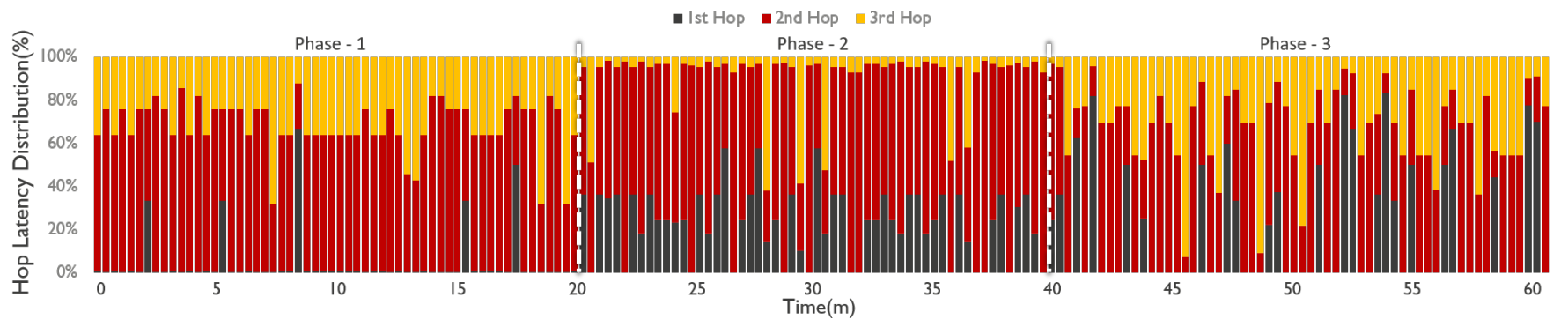

(c) The Share of Each Hop in Total Latency.

Fig. 10: Per-Packet and Per-Hop Packet Loss Rate and Latency Measurement Results. Phase 1: Manageable traffic from Node 4 to Node 6. Phase 2: Extra traffic from Node 5 and 2 towards BR. Phase 3: Node 4 traffic rate is increased to a level where the network can not handle.

The performance trends in these figures show that the AMPM was able to capture the performance deviation between different phases. In the initial phase, the network was able to provide reliable and latency-bounded performance with nearzero packet loss rate (on average $0.2 \%$ ) and low delay values (on average 31.48 slots). When non-critical traffic is generated across the network, although the network performance was slightly affected, it was still able to provide sufficient communication performance (on average $0.6 \%$ loss rate and 31.50 slots latency). While, in this case, it is clear that the share of the first and second hop in the total delay is remarkably increased which demonstrates the increase in the occupation rate of the corresponding nodes due to non-critical traffic. Concerning the last phase, where the rate of the critical traffic is slightly increased, the critical application traffic starts to face performance drops and consequently the packet loss rate (on average 6.0\%) and latency (on average 66 slots) values escalate and become unstable.

Since these measurement results collected via AM-PM in simulation and experimental tests are parallel to what could be expected and since AM-PM-based telemetry solution is able to capture the changes in the network performance due to various reasons, these tests confirm that AM-PM can be used to realize accurate loss and delay measurements over IWSNs.

\begin{tabular}{|l|r|r|r|r|r|r|}
\cline { 2 - 8 } \multicolumn{1}{c|}{} & \multicolumn{3}{c|}{ Average } & \multicolumn{3}{c|}{ Maximum } \\
\cline { 2 - 8 } \multicolumn{1}{c|}{} & Phase1 & Phase2 & \multicolumn{1}{c|}{ Phase3 } & \multicolumn{1}{c|}{ Phase1 } & \multicolumn{1}{c|}{ Phase2 } & \multicolumn{1}{c|}{ Phase3 } \\
\hline Delay & 31.48 & 31.50 & 66.00 & 66.00 & 77.00 & 374.00 \\
\hline Loss Rate & $0.2 \%$ & $0.6 \%$ & $6.0 \%$ & $4.3 \%$ & $8.0 \%$ & $31.4 \%$ \\
\hline
\end{tabular}

Fig. 11: End-to-End Delay and Packet Loss Measurement Statistics.

\begin{tabular}{|c|c|r|r|r|r|r|r|}
\cline { 3 - 9 } \multicolumn{2}{c|}{} & \multicolumn{3}{c|}{ Average } & \multicolumn{3}{c|}{ Maximum } \\
\cline { 3 - 9 } \multicolumn{2}{c|}{} & Phase1 & Phase2 & Phase3 & Phase1 & Phase2 & \multicolumn{1}{c|}{ Phase3 } \\
\hline \multirow{3}{*}{ Delay } & 1st Hop & 1.89 & 7.07 & 20.29 & 44.00 & 19.00 & 198.00 \\
\cline { 2 - 9 } & 2nd Hop & 19.88 & 20.59 & 23.38 & 47.00 & 57.00 & 56.00 \\
\cline { 2 - 9 } & 3rd Hop & 9.71 & 3.84 & 22.33 & 30.00 & 45.00 & 340.00 \\
\hline \multirow{3}{*}{ Loss Rate } & 1st Hop & $0.0 \%$ & $0.0 \%$ & $2.4 \%$ & $0.0 \%$ & $0.0 \%$ & $28.6 \%$ \\
\cline { 2 - 8 } & 2nd Hop & $0.1 \%$ & $0.4 \%$ & $1.4 \%$ & $3.6 \%$ & $7.4 \%$ & $8.3 \%$ \\
\cline { 2 - 8 } & 3rd Hop & $0.1 \%$ & $0.2 \%$ & $2.3 \%$ & $3.8 \%$ & $8.0 \%$ & $18.2 \%$ \\
\hline
\end{tabular}

Fig. 12: Hop-by-Hop Delay and Packet Loss Measurement Statistics.

\section{DisCUSSION}

First of all, the proposed AM-PM mechanism in IWSNs creates an efficient and light-weight monitoring solution, which brings no extra overhead on the monitored packets and requires very limited memory utilization and negligible computational effort. It also does not require any dedicated middle-boxes or 
measurement devices. However, it requires a limited amount of extra resource usage for reporting the collected telemetry data to Network Manager. But, this extra resource usage can be eliminated or minimized by combining with other health and status reporting messages or by using resources that are not dedicated to critical communication.

Secondly, the AM-PM mechanism is offering a robust, reliable and accurate measurement solution for IWSNs by assuring fate-sharing (in other words, the packets used for measuring the network are sharing the fate of the data packets), achieving single packet loss granularity and tolerating the out-of-order packet delivery. It also provides fine-grained telemetry data by collecting per-flow, per-hop and per-packet performance measurements.

Additionally, the resulting system design offers a flexible, self-organizing and distributed telemetry solution by enabling each node to decide which flows to monitor and with which frequency, also to determine which packets to monitor for delay performance.

Since it does not require any change in the utilized protocols or packet fields, it does not have any interoperability issue with existing devices and systems.

Therefore, the proposed 6TiSCH AM-PM solution has great potential to be used for achieving maximized visibility and advanced manageability in various network elements and application scenarios. For instance, based on end-to-end latency and reliability measurements, QoS Validation and Service Verification can be achieved. The per-hop telemetry data (transfer delay, etc.) can be used for Debugging \& Troubleshooting or Link Utilization Monitoring and Congestion Control.

\section{CONCLUSION}

In this paper, the problem of efficient and accurate network monitoring in Industrial WSNs is addressed, mainly targeting critical application traffic. Particularly, a conceptual design for an Alternate Marking network monitoring mechanism for IEEE 802.15.4e TSCH technologies, more specifically 6TiSCH Networks, is presented, which was inspired by the recent AM-PM concept.

By means of implementation, simulations and testbed measurements, it is demonstrated that the proposed AM-PM approach in IWSNs creates an efficient, accurate, reliable and flexible monitoring solution that can provide flow-based perhop and per-packet telemetry data, validating its suitability for Industrial WSNs. Although this paper mainly describes and implements the proposed AM-PM solution for the $6 \mathrm{TiSCH}$ protocol stack, the same design can be also applied to any $802.15 .4 \mathrm{e}-$-like and TSCH-based networking technologies; such as WirelessHart and ISA100.11a.

The proposed AM-PM mechanism can enable performing a wide range of monitoring and management operations in various network scenarios, especially for verifying Quality of Service (QoS) for the critical application traffic. Besides stand-alone usage, the proposed AM-PM mechanism can be also deployed alongside other existing out-of-band and in-band monitoring mechanisms and complement them by providing detailed and accurate measurement information and timely available per-flow and per-hop telemetry data.

\section{ACKNOWLEDGMENT}

This work was partially funded by the FWO-Flanders, under grant agreement \#G055619N. Part of this research was funded by the ICON project Internet of Shipping (IoS). IoS is realized in collaboration with imec, with project support from VLAIO. Project partners are imec, Exmar, Ovinto and Aloxy.

\section{REFERENCES}

[1] IEC-62591, "Industrial communication networks - Wireless communication network and communication profiles-WirelessHART."

[2] IEC-62734, "Industrial networks - Wireless communication network and communication profiles - ISA 100.11a."

[3] "IEEE standard for local and metropolitan area networks-part. 15.4: low-rate wireless personal area networks (LR-WPANs) amendment 1: MAC sublayer,' IEEE, Standard 802.15.4e-2012, Apr. 2012.

[4] P. Thubert, Ed., "An architecture for IPv6 over the TSCH mode of IEEE 802.15.4," IETF, Internet-Draft draft-ietf-6tisch-architecture-24, July 2019.

[5] D. Raposo et al., "Industrial IoT monitoring: Technologies and architecture proposal," Sensors, vol. 18, no. 10, p. 3568, Oct 2018.

[6] T. Mizrahi, V. Vovnoboy, M. Nisim, G. Navon, and A. Soffer, "Network telemetry solutions for data center and enterprise networks," Marvell, White Paper, March 2018.

[7] The P4.org Applications Working Group, "In-band network telemetry (INT) dataplane specification," The P4 Language Consortium, Tech. Rep., August 2018.

[8] A. Riesenberg et al., "Time-multiplexed parsing in marking-based network telemetry," in Proceedings of the 12th ACM International Conference on Systems and Storage (SYSTOR), 2019.

[9] G. Fioccola et al., "Alternate-marking method for passive and hybrid performance monitoring," IETF, RFC 8321, Jan 2018.

[10] X. Vilajosana, T. Watteyne, M. Vučinić, T. Chang, and K. S. J. Pister, "6tisch: Industrial performance for ipv6 internet-of-things networks," Proceedings of the IEEE, vol. 107, no. 6, pp. 1153-1165, June 2019.

[11] A. Karaagac, J. Haxhibeqiri, I. Moerman, and J. Hoebeke, "Timecritical communication in 6tisch networks," in 2018 IEEE Wireless Communications and Networking Conference Workshops (WCNCW), April 2018, pp. 161-166.

[12] A. Karaagac, I. Moerman, and J. Hoebeke, "Hybrid schedule management in 6TiSCH networks: The coexistence of determinism and flexibility," IEEE Access, vol. 6, pp. 33 941-33 952, 2018.

[13] M. Veillette et al., "CoAP management interface," IETF, Internet-Draft draft-ietf-core-comi-07, July 2019.

[14] F. Brockners et al., "Requirements for in-situ OAM," IETF, InternetDraft draft-brockners-inband-oam-requirements-03, March 2017.

[15] A. Karaagac, E. De Poorter, and J. Hoebeke, "In-band network telemetry in industrial wireless sensor networks," IEEE Transactions on Network and Service Management, pp. 1-1, 2019.

[16] M. Cociglio et al., "A packet-based method for passive performance monitoring," IETF, Internet-Draft draft-tempia-opsawg-p3m-00, March 2011.

[17] T. Mizrahi et al., "AM-PM: Efficient network telemetry using alternate marking," IEEE Network, vol. 33, no. 4, pp. 155-161, July 2019.

[18] T. Mizrahi et al., "Compact alternate marking methods for passive and hybrid performance monitoring," IETF, Internet-Draft draft-mizrahiippm-compact-alternate-marking-03, October 2018.

[19] Huawei Technologies, "Huawei agile campus network iPCA solution," Technology White Paper, December 2014

[20] "IEEE standard for low-rate wireless networks," IEEE, Standard 802.15.4-2015, 2015

[21] S. Duquennoy et al. Contiki-NG: The OS for next generation IoT devices. [Online]. Available: https://github.com/contiki-ng/contiki-ng

[22] S. Duquennoy, A. Elsts, B. A. Nahas, and G. Oikonomo, "TSCH and 6TiSCH for Contiki: Challenges, design and evaluation," in Proc. of the 13th International Conference on Distributed Computing in Sensor Systems (DCOSS), June 2017.

[23] Wireless testlab and officelab. [Online]. Available: https://doc.ilabt.imec.be/ilabt/wilab/ 